\title{
THE QUASIREGULAR PROJECTIVE PLANES OF ORDER
}

16

\author{
MARC RÖDER \\ National University of Ireland, Galway
}

\begin{abstract}
The projective planes of order 16 admitting a large $(\geq$ 137) quasiregular group of collineations are classified. The classification is done using the theorem of Dembowski and Piper [DP67] and a complete search by computer. No new planes are found.
\end{abstract}

\section{Introduction}

Let $\mathfrak{P}$ be a projective plane of order $n$ and $G$ a group of collineations of $\mathfrak{P}$. The group $G$ is called quasiregular if it acts regularly on its point and line orbits, i.e. if the point-(line-)stabiliser is a normal subgroup in $G$. The quasiregular group is called large if $|G|>\frac{1}{2}\left(n^{2}+n+1\right)$. By [Dem68, 4.2.8, p.181], a group of collineations of a projective plane acts faithfully on at least one point or line orbit. Up to duality, we may assume that this is an orbit of points. So a large quasiregular group of collineations has exactly one orbit of size $|G|$ on points. One has:

Theorem 1.1 (Dembowski-Piper, [DP67], [Dem68, 4.2.10, p.182]). Let $G$ be a quasiregular group of collineations of the projective plane $\mathfrak{P}$ of order $n$. Denote by $m=m(G)$ the number of point (or line) orbits of $G$, and by $\mathbf{F}=\mathbf{F}(G)$ the substructure of the elements fixed by $G$. If $|G|>\frac{1}{2}\left(n^{2}+n+1\right)$, then there are only the following possibilities:

$\mathbf{D P}_{a}|G|=n^{2}+n+1, m=1$ and $\mathbf{F}=\emptyset$. Here $G$ is transitive.

$\mathbf{D P}_{b}|G|=n^{2}, m=3$ and $\mathbf{F}$ is a flag.

$\mathbf{D P}_{c}|G|=n^{2}, m=n+2$ and $\mathbf{F}$ is either a unique line $A$ and all $x \in A$ or dually a unique point $c$ and all lines $X \in[c]$.

2000 Mathematics Subject Classification. 05B10, 05B25.

Key words and phrases. Projective planes, relative difference sets, quasiregular group of collineations, complete computer search. 
$\mathbf{D P}_{d}|G|=n^{2}-1, m=3$ and $\mathbf{F}$ is a unique non-incident point-line pair.

$\mathbf{D P}_{e}|G|=n^{2}-\sqrt{n}, m=2$ and $\mathbf{F}=\emptyset$. In this case, one point and one line orbit together form a Baer subplane.

$\mathbf{D P}_{f}|G|=n(n-1), m=5$ and $\mathbf{F}$ consists of two points $u, v$, the line containing $u$ and $v$ and one other line through one of $u, v$.

DP $_{g}|G|=(n-1)^{2}, m=7$ and $\mathbf{F}$ consists of the vertices and sides of a triangle.

In the following we will describe a computer search which leads to the classification result of Theorem 1.2.

TheOREM 1.2. Let $\mathfrak{P}$ be a projective plane of order 16 with a large quasiregular collineation group $G$ and a regular point orbit. Then $\mathfrak{P}$ is a translation plane or the Mathon plane. Moreover the desarguesian plane occurs as types $\mathbf{D} \mathbf{P}_{a}, \mathbf{D P}_{b}, \mathbf{D P}_{c}, \mathbf{D P}_{d}, \mathbf{D P}_{f}$ or $\mathbf{D} \mathbf{P}_{g}$. The two semifield planes occur as types $\mathbf{D P}_{b}$ or $\mathbf{D} \mathbf{P}_{c}$. The remaining translation planes occur only as type $\mathbf{D} \mathbf{P}_{c}$ and the Mathon plane has type $\mathbf{D} \mathbf{P}_{b}$.

The translation planes of order 16 have been classified in [DR83], i.e. only types different from $\mathbf{D P} \mathbf{P}_{c}$ have to be considered. In each of these cases, regular orbits on points and lines exist. This leads to the difference set construction which we describe in the next section.

A complete list of all groups and the types of planes they act on is given in the appendix.

\section{Notation, Relative Difference SETS}

Let $G$ be a finite group and $D, N \subseteq G$. We say that $D$ is a relative difference set with forbidden set $N$, if there exists $0<\lambda \in \mathbb{Z}$ such that every element of $G-N$ can be expressed in exactly $\lambda$ ways as a quotient $d_{1} d_{2}^{-1}$ with $d_{1}, d_{2} \in D$ and no element of $N-1$ can be written like this. In group ring notation: $D D^{(-1)}=k+\lambda(G-N)$.

We call $(|G| /|N|,|N|, k, \lambda)$ with $k=|D|$ the parameter tuple of the difference set $D$.

A relative difference set is called ordinary difference set or simply difference set if $N=1$ holds. The incidence structure $\operatorname{dev} D:=(G,\{D g \mid g \in$ $G\}, \in$ ) is called development of $D$ and $G$ acts (via right multiplication) as a regular group of collineations on $\operatorname{dev} D$.

A set $D \subset G$ is called partial relative difference set with forbidden set $N \subseteq G$, if every element outside $N$ can be written in at most $\lambda$ ways as a quotient in $D$ and no element of $N-1$ is a quotient of two elements of $D$.

Two partial relative difference sets $D, D^{\prime} \subseteq G$ are called equivalent if there is a $g \in G$ and $\phi \in \operatorname{Aut}(G)$ such that $D=(D g)^{\phi}$. Two partial relative difference sets $D, D^{\prime} \subseteq G$ are called strongly equivalent if they are equivalent and have the same forbidden set. 
TABLE 1. The difference set parameters of Theorem 1.1

\begin{tabular}{c|c|c} 
type & $(|G| /|N|,|N|, k, \lambda)$ & \\
\hline $\mathbf{D P}_{a}$ & $\left(n^{2}+n+1,1, n+1,1\right)$ & $N=1$ \\
$\mathbf{D P}_{b}$ & $(n, n, n, 1)$ & $N \unlhd G$ \\
$\mathbf{D P}_{d}$ & $(n+1, n-1, n, 1)$ & $N \unlhd G$ \\
$\mathbf{D P}_{e}$ & $(n+\sqrt{n}+1, n-\sqrt{n}, n-1,1)$ & $N=A \cup B, A, B \unlhd G$ \\
$\mathbf{D P}_{f}$ & $(n / 2,2 n-2, n-1,1)$ & $|B|+1=|A|=n, A \times B=G$ \\
& & $N=A \cup B \cup C, A \times B=G$ \\
$\mathbf{D P}_{g}$ & $\left((n-1)^{2} / 3 n-5,3 n-5, n-1,1\right)$ & $|A|=|B|=|C|=n-1$
\end{tabular}

Obviously, equivalent relative difference sets induce isomorphic developments. Note that, in general $\operatorname{dev} D^{-1} \nsim \operatorname{dev} D$. Instead, it is easy to see that $\operatorname{dev} D^{-1} \sim(\operatorname{dev} D)^{d}$, where ${ }^{d}$ denotes the dual incidence structure.

For more about relative difference sets see [Pot95, Sch02, GJ03].

2.1. Difference sets from projective planes. Assume now that $\mathfrak{P}$ is a projective plane of order $n$ and $G$ a large quasiregular group of collineations which has a regular point and line orbit. We identify the elements in that point orbit with the elements of $G$. Let $D$ be the intersection of the regular point orbit with a line from the regular line orbit. Then $D$ is a relative difference set. In table 1 , the parameters for the difference sets of the Dembowski-Piper theorem are given. The last column contains a description of the forbidden set. Observe that in all cases, $N$ is either a normal subgroup of $G$ or a union of normal subgroups. As $\mathbf{D} \mathbf{P}_{c}$ defines translation planes, it does not have a description in terms of relative difference sets.

It is not difficult to see that in all cases $\operatorname{dev} D$ can be extended to a projective plane of order $n$ and that this extension is uniquely determined. So in order to obtain Theorem 1.2 we have to classify all relative difference sets of the respective types for $n=16$.

\section{THE SEARCH}

Before we give an algorithm to calculate all relative difference sets for each of the cases of Theorem 1.1, we introduce an invariant for partial relative difference sets which will be used during the search.

3.1. An invariant for partial relative difference sets. The following lemma is a generalisation of a lemma of Bruck [Bru55] and the proof is very similar to the original one.

Lemma 3.1. Let $G$ be a finite group and $U \unlhd G$. Furthermore, let $D \subseteq G$ be a relative difference set with forbidden set $N$. We define $v_{i}:=\left|D \cap g_{i}\right|$, where $\left\{g_{1}, \ldots, g_{|G: U|}\right\}=G / U=G^{\rho}$ and $\rho$ is the natural homomorphism. Let $g_{1}=U$ and $v_{i j}=\left|D \cap g_{i} g_{j}\right|$. Then 
1. $\sum v_{i}=k$.

2. $\sum v_{i}^{2}=\lambda(|U|-|U \cap N|)+k$.

3. $\sum_{j} v_{j} v_{i j}=\lambda\left(|U|-\left|g_{i} \cap N\right|\right)$ for $i \neq 1$.

4. If $N \leq G$, then

$$
\sum_{j} v_{j} v_{i j}= \begin{cases}\lambda(|U|-|U \cap N|), & \text { if } g_{1} \neq g_{i} \in N^{\rho}, \\ \lambda|U|, & \text { if } g_{i} \notin N^{\rho} .\end{cases}
$$

Note that $v_{i j} \in\left\{v_{1}, \ldots, v_{|G: U|}\right\}$ is just the coefficient of $g_{i} g_{j} \in G / U$ in $D^{\rho} \in \mathbb{Z}[G / U]$. As the right sides in equations 1.-4. are known, we may calculate solutions $\left(v_{1}, \ldots, v_{|G: U|}\right)$ of these equations before we search for a difference set $D$. This results in a significant reduction of the search space.

Definition 3.2. Let $N, U, G$ be as in Lemma 3.1. Let $G / U=$ $\left\{g_{1}, \ldots, g_{|G: U|}\right\}$ with $g_{1}=U$ and $v=\left(v_{1}, \ldots, v_{|G: U|}\right)$ a solution of 1.-4. (with $\left.v_{i j}=v_{g_{i} g_{j}}\right)$. Then $v$ is called ordered signature for $U$ (relative to $\left.N\right)$. The corresponding multiset $\|v\|$ is called admissible signature for $U$ (relative to $N$ ). Moreover we define a mapping $s_{U}: \mathcal{P}(G) \rightarrow \mathbb{N}^{|G: U|}$ by $s_{U}(S)(i)=\left|S \cap g_{i}\right|$ and denote by $\sigma_{U}(S)$ the multiset $\left\|s_{U}(S)\right\|$.

For every relative difference set $D$ and every $U \unlhd G$, the tuple $s_{U}(D)$ is an ordered signature and the multiset $\sigma_{U}(D)$ is an admissible signature.

It is obvious that if $\mathcal{U}$ is a system of representatives of the $\operatorname{Aut}(G)$-orbits on $\{U \unlhd G|| U \mid \in \mathcal{I}\}$ for some $\mathcal{I} \subseteq \mathbb{N}$, and $S$ a partial relative difference set, we have

$$
\left\|\left(\sigma_{U}(S)\right)_{U \in \mathcal{U}}\right\|=\left\|\left(\sigma_{U}\left((S g)^{\phi}\right)\right)_{U \in \mathcal{U}}\right\| \text { for all } g \in G \text { and all } \phi \in \operatorname{Aut}(G) .
$$

So the mappings $\sigma_{U}$ can be used as an invariant for equivalence classes of partial relative difference sets. And we may in some cases even be able to decide that a partial relative difference set can not be extended to a full relative difference set by comparing its image under $\sigma_{U}$ to admissible signatures. Note that using $\operatorname{Aut}(G)_{N}$ instead of $\operatorname{Aut}(G)$ gives an invariant for strong equivalence.

3.2. The algorithm. We will now outline the algorithm used in the search for quasiregular projective planes of order 16. For each case of Theorem 1.1 other than $\mathbf{D P} \mathbf{P}_{c}$, all possible groups and the respective forbidden sets are calculated using the small groups library provided by GAP. Restrictions on the forbidden sets are given in table 1 . For each group $G$ and all possible forbidden sets $N$, relative difference sets are then constructed using the following method:

1. choose a set $\mathcal{V}$ of normal subgroups of $G$ (representatives of $\operatorname{Aut}(G)_{N}$ orbits, for instance) and calculate admissible signatures for them

2. choose normal subgroup $U \notin \mathcal{V}$ of small index with "nice" admissible signatures 
3. generate partial relative difference sets (of maximal length) in $U$. Use the mappings $\sigma_{V}$ with $V \in \mathcal{V}$ to partition the found partial relative difference sets and a test for strong equivalence on the partition elements

4. continue generating partial difference sets by adding elements from non-trivial cosets modulo $U$ as above (coset by coset) or by brute force search (omitting partitioning and equivalence tests)

5. generate projective planes from the relative difference sets found

6. run isomorphism test on planes

The algorithm was implemented for each of the relevant cases of Theorem 1.1. Depending on the special case, slightly different implementations were used for the above steps. For example, equivalence tests can be performed at step 3. before the maximal length of partial relative difference sets in $U$ is reached. Some further aspects of the implementation will be discussed in the next section. A more detailed description is given in [Röd06a].

3.3. Remarks concerning implementation. The calculation was done using the computer algebra system GAP [GAP] and a package especially written for this purpose [Röd06b]. GAP's small groups library is used to look up all possible groups. Implementations are available at [Röd].

3.3.1. Signatures and "nice" normal subgroups. For normal subgroups of low index, it is possible to calculate not only admissible, but even ordered signatures. Subgroups for which ordered signatures are known are considered "nice" for step 2. of the algorithm. Of particular interest are those subgroups which do only have one ordered signature (up to permutations induced by right multiplication with group elements).

In case $\mathbf{D P}_{a}$, we also calculated ordered signatures for normal subgroups of larger index using basic representation arguments for some groups $G$ (see [Röd06a] for details). Knowing ordered signatures enables us to use the mappings $s_{V}$ for some $V$ in step 3. This is much more restrictive than using just admissible signatures and $\sigma_{V}$.

3.4. Special methods for case $\mathbf{D} \mathbf{P}_{b}$. In case $\mathbf{D} \mathbf{P}_{b}$, all groups of order $2^{8}$ have to be considered. A preliminary argument is used to reduce the number of groups in which a search for relative difference sets is actually conducted.

LEMma 3.3. Let $D \subseteq G$ be a relative difference set of order $n \equiv 0 \bmod 2$ and type $\mathbf{D P}_{b}$ with forbidden subgroup $N \unlhd G$. Let $\iota \in G$ be an involution. Then $\iota \in N$.

Proof. Let $\mathfrak{P}$ be a projective plane of type $\mathbf{D P}_{b}$ and order $n$. Let $L_{\infty}$ the line fixed by $G$. Any involutorial collineation of $\mathfrak{P}$ is either planar or an elation [Dem68, 4.1.9].

The affine points, i.e. the points not on $L_{\infty}$ form a regular orbit and are identified with the elemets of $G$. Let $\iota \in G$ be an involution. Then $\iota$ is not 
planar as $\iota$ has no affine fixed-point. Thus $\iota$ is an elation. Then $\iota$ fixes $n$ affine lines which must be the cosets of $N$ as $\iota$ acts fixed-point-freely on the affine lines $\{D g \mid g \in G\}$. Hence $N \iota=N$ and thus $\iota \in N$.

In particular, a relative difference set of type $\mathbf{D} \mathbf{P}_{b}$ cannot exist in a group containing "too many" involutions. This also rules out some subgroups as forbidden subgroups (namely those not containing all involutions of the full group).

3.4.1. Invariants for full projective planes. Isomorphism tests are done using the number of Fano-subplanes as an invariant of projective planes. For type $\mathbf{D} \mathbf{P}_{b}$, the group of translations is calculated to determine if a plane is a translation plane. By [Roy], the translation planes of order 16 are uniquely determined by the number of Fano-subplanes (all translation planes of order 16 are known by [DR83]). For the remaining case, explicit isomorphisms are constructed. The data for the known planes is available from [Roy].

ACKNOWLEDGEMENTS.

The author would like to thank U. Dempwolff for his support and for independently verifying the result of case $\mathbf{D} \mathbf{P}_{b}$.

\section{Appendix: Detailed Results}

This appendix lists all groups that admit an action as a large quasiregular group of collineations on a projective plane of order 16. Because of its size the case $\mathbf{D} \mathbf{P}_{b}$ will be treated in a separate section below.

4.1. Cases $\mathbf{D} \mathbf{P}_{a}-\mathbf{D} \mathbf{P}_{g}$ without $\mathbf{D} \mathbf{P}_{b}$. Let $\mathfrak{P}$ be a projective plane of order 16 and $G$ a group acting on $\mathfrak{P}$ as a large quasiregular group of collineations. If $\mathfrak{P}$ is of type

$\mathbf{D P}_{a}$ then $G \simeq C_{273}$ or $G \simeq C_{3} \ltimes C_{91} \simeq\left\langle a, b|| a|=3| b \mid,=91, b^{a}=b^{16}\right\rangle$. Note that there are two isomorphism types of groups $C_{3} \ltimes C_{91}$, but only one admits an action of type $\mathbf{D} \mathbf{P}_{a}$.

$\mathbf{D P}_{d}$ then $G \simeq C_{255}$.

$\mathbf{D P}_{f}$ then $G \simeq C_{15} \times C_{2}^{4}$.

$\mathbf{D P}_{g}$ then $G \simeq C_{15}^{2}$.

In each of these cases, $\mathfrak{P}$ is the Desarguesian plane.

There is no projective plane of type $\mathbf{D P}_{e}$ and order 16 . The planes of type $\mathbf{D} \mathbf{P}_{c}$ are translation planes. For their classification we refer the reader to [DR83].

4.2. Case $\mathbf{D} \mathbf{P}_{b}$. The following table contains the 590 groups of order 256 which admit relative difference sets of type $\mathbf{D P}_{b}$.

The column labels "Des", "Semi 2", "Semi 4" and "Mathon" stand for the Desarguesian plane, the semifield planes with kernel 2 and 4, respectively, and the Mathon plane. Note that a group acts on the Mathon plane if and 
only if it acts on the dual Mathon plane. So there is no extra column for the dual Mathon plane. And, of course, a mark means that there is an action on the corresponding plane. Groups which are not in the table do not admit a relative difference set of type $\mathbf{D} \mathbf{P}_{b}$.

The groups are numbered as in the small groups library of GAP:

There are 56092 groups of order 256.

They are sorted by their ranks.

1 is cyclic.

2 - 541 have rank 2.

542 - 6731 have rank 3.

6732 - 26972 have rank 4.

26973 - 55625 have rank 5.

55626 - 56081 have rank 6.

56082 - 56091 have rank 7.

56092 is elementary abelian.

Group number 6732 is $C_{4}^{4}$.

\begin{tabular}{|c|c|c|c|c|}
\hline Nr. & $\stackrel{\mathscr{0}}{0}$ & 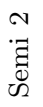 & 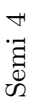 & $\begin{array}{l}\frac{0}{7} \\
\stackrel{\sigma}{\pi}\end{array}$ \\
\hline 296 & $\bullet$ & $\bullet$ & $\bullet$ & \\
\hline 331 & $\bullet$ & & & $\bullet$ \\
\hline 420 & & & & - \\
\hline 843 & & $\bullet$ & $\bullet$ & - \\
\hline 855 & $\bullet$ & $\bullet$ & & \\
\hline 874 & & & & - \\
\hline 876 & & & & $\bullet$ \\
\hline 909 & & & & $\bullet$ \\
\hline 938 & & $\bullet$ & $\bullet$ & \\
\hline 947 & $\bullet$ & $\bullet$ & & \\
\hline 956 & & & & - \\
\hline 961 & & & & $\bullet$ \\
\hline 963 & & $\bullet$ & $\bullet$ & \\
\hline 978 & & & & $\bullet$ \\
\hline 980 & & & & $\bullet$ \\
\hline 985 & & & & $\bullet$ \\
\hline 1001 & & & & $\bullet$ \\
\hline 1038 & & & & $\bullet$ \\
\hline 1052 & & & & $\bullet$ \\
\hline 1053 & & & & $\bullet$ \\
\hline 1060 & & $\bullet$ & $\bullet$ & \\
\hline 1066 & & & & $\bullet$ \\
\hline 1081 & & & & $\bullet$ \\
\hline 1086 & & $\bullet$ & $\bullet$ & \\
\hline 1101 & & & & $\bullet$ \\
\hline 1104 & & & & $\bullet$ \\
\hline 1108 & & & & $\bullet$ \\
\hline 3322 & & & & $\bullet$ \\
\hline
\end{tabular}

\begin{tabular}{|c|c|c|c|c|}
\hline Nr. & $\stackrel{\tilde{0}}{0}$ & & $\begin{array}{l}\vec{H} \\
\text { "च्च } \\
\text { W }\end{array}$ & $\mid \begin{array}{l}5 \\
0 \\
0\end{array}$ \\
\hline$\overline{4509}$ & $\bullet$ & & & $\bullet$ \\
\hline 5287 & & & & 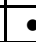 \\
\hline 5688 & & $\bullet$ & $\bullet$ & \\
\hline 5848 & $\bullet$ & $\bullet$ & $\bullet$ & \\
\hline 6732 & $\bullet$ & & & \\
\hline 6738 & & & $\bullet$ & \\
\hline 6753 & & $\bullet$ & & \\
\hline 6756 & & $\bullet$ & & \\
\hline 6760 & & $\bullet$ & & \\
\hline 6769 & & $\bullet$ & & \\
\hline 6774 & & & $\bullet$ & \\
\hline 6775 & & $\bullet$ & & \\
\hline 6781 & & $\bullet$ & $\bullet$ & \\
\hline 6785 & & $\bullet$ & & \\
\hline 6792 & & $\bullet$ & & \\
\hline 6794 & & & $\bullet$ & \\
\hline 6800 & & $\bullet$ & & \\
\hline 6807 & & $\bullet$ & & \\
\hline 6814 & & $\bullet$ & $\bullet$ & \\
\hline 6817 & $\bullet$ & & & \\
\hline 6821 & & $\bullet$ & $\bullet$ & \\
\hline 6822 & & $\bullet$ & & \\
\hline 6838 & & & $\bullet$ & \\
\hline 6842 & & $\bullet$ & $\bullet$ & \\
\hline 6843 & & $\bullet$ & & \\
\hline 6844 & & $\bullet$ & & \\
\hline 6848 & & $\bullet$ & $\bullet$ & \\
\hline 6851 & & $\bullet$ & & \\
\hline
\end{tabular}

\begin{tabular}{|c|c|c|c|c|}
\hline Nr. & $\stackrel{0}{0}$ & 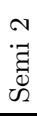 & 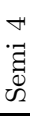 & \\
\hline 6852 & & - & & \\
\hline 6873 & & • & & \\
\hline 6897 & & • & & \\
\hline 6916 & & $\bullet$ & & \\
\hline 6917 & & - & & \\
\hline 6919 & & - & $\bullet$ & \\
\hline 6920 & & $\bullet$ & & \\
\hline 6922 & & $\bullet$ & & \\
\hline 6938 & & $\bullet$ & $\bullet$ & \\
\hline 6942 & & - & & \\
\hline 6943 & & - & & \\
\hline 6949 & & $\bullet$ & • & \\
\hline 6952 & & $\bullet$ & $\bullet$ & \\
\hline 6964 & & $\bullet$ & & \\
\hline 6966 & & $\bullet$ & & \\
\hline 6973 & & $\bullet$ & & \\
\hline 6988 & & $\bullet$ & & \\
\hline 6991 & & & $\bullet$ & \\
\hline 6994 & & $\bullet$ & & \\
\hline 6997 & & $\bullet$ & & \\
\hline 7012 & & $\bullet$ & - & \\
\hline 7030 & & - & & \\
\hline 7036 & & $\bullet$ & & \\
\hline 7039 & & $\bullet$ & & \\
\hline 7043 & & $\bullet$ & & \\
\hline 7045 & & $\bullet$ & & \\
\hline 7046 & & & $\bullet$ & \\
\hline 7048 & & - & & \\
\hline
\end{tabular}


M. RÖDER

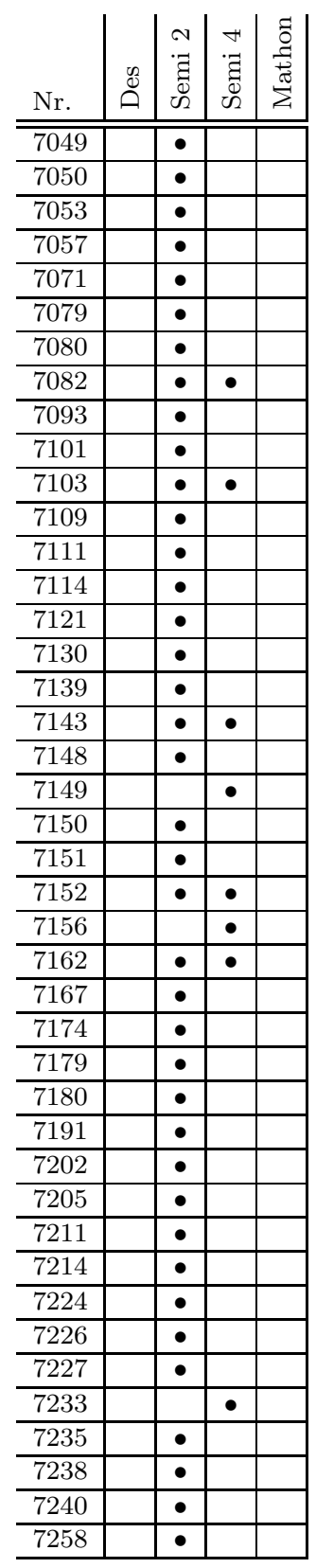

\begin{tabular}{|c|c|c|c|c|}
\hline Nr. & $\stackrel{\omega}{0}^{\infty}$ & $\stackrel{N}{\because}$ & $\begin{array}{l}+ \\
\vec{\Xi} \\
\text { "n }\end{array}$ & \\
\hline$\overline{7268}$ & & $\bullet$ & & \\
\hline 7272 & & & $\bullet$ & \\
\hline 7274 & & $\bullet$ & & \\
\hline 7284 & & $\bullet$ & & \\
\hline 7296 & & $\bullet$ & & \\
\hline 7306 & & • & & \\
\hline 7308 & & $\bullet$ & & \\
\hline 7316 & & $\bullet$ & & \\
\hline 7318 & & $\bullet$ & & \\
\hline 7334 & & $\bullet$ & & \\
\hline 7344 & & $\bullet$ & & \\
\hline 7366 & & & $\bullet$ & \\
\hline 7382 & & $\bullet$ & & \\
\hline 7402 & & $\bullet$ & & \\
\hline 7423 & & $\bullet$ & & \\
\hline 7429 & $\bullet$ & & & \\
\hline 7438 & & $\bullet$ & $\bullet$ & \\
\hline 7446 & & $\bullet$ & & \\
\hline 7447 & & $\bullet$ & & \\
\hline 7453 & & $\bullet$ & & \\
\hline 7454 & & $\bullet$ & $\bullet$ & \\
\hline 7458 & & $\bullet$ & & \\
\hline 7459 & $\bullet$ & $\bullet$ & $\bullet$ & \\
\hline 7460 & & $\bullet$ & & \\
\hline 7465 & & $\bullet$ & & \\
\hline 7471 & & $\bullet$ & $\bullet$ & \\
\hline 7473 & & $\bullet$ & & \\
\hline 7477 & & $\bullet$ & $\bullet$ & \\
\hline 7478 & & $\bullet$ & & \\
\hline 7489 & & $\bullet$ & & \\
\hline 7490 & & $\bullet$ & & \\
\hline 7498 & $\bullet$ & & & \\
\hline 7499 & & $\bullet$ & & \\
\hline 7519 & & $\bullet$ & & \\
\hline 7526 & & & $\bullet$ & \\
\hline 7538 & & $\bullet$ & & \\
\hline 7540 & & $\bullet$ & & \\
\hline 7542 & & $\bullet$ & & \\
\hline 7549 & & $\bullet$ & & \\
\hline 7562 & $\bullet$ & & & \\
\hline 7581 & & $\bullet$ & & \\
\hline 7583 & & $\bullet$ & & \\
\hline
\end{tabular}

\begin{tabular}{|c|c|c|c|}
\hline Nr. & $\stackrel{0}{0}$ & $\begin{array}{l}\text { N } \\
\ddot{\Xi} \\
\stackrel{0}{0}\end{array}$ & $\begin{array}{l}\vec{H} \\
\cdot \vec{\Xi} \\
\stackrel{\nu}{ } \\
ٌ\end{array}$ \\
\hline$\overline{7585}$ & & $\bullet$ & \\
\hline 7586 & & $\bullet$ & \\
\hline 7587 & & $\bullet$ & $\bullet$ \\
\hline 7589 & & - & \\
\hline 7593 & & $\bullet$ & \\
\hline 7596 & & & $\bullet$ \\
\hline 7602 & & & $\bullet$ \\
\hline 7622 & & $\bullet$ & $\bullet$ \\
\hline 7626 & & $\bullet$ & $\bullet$ \\
\hline 7636 & & $\bullet$ & \\
\hline 7638 & & $\bullet$ & \\
\hline 7651 & & & $\bullet$ \\
\hline 7652 & $\bullet$ & & \\
\hline 7656 & & $\bullet$ & $\bullet$ \\
\hline 7691 & $\bullet$ & $\bullet$ & \\
\hline 7697 & $\bullet$ & & \\
\hline 7698 & & $\bullet$ & \\
\hline 7767 & & $\bullet$ & \\
\hline 7769 & & $\bullet$ & \\
\hline 7775 & & $\bullet$ & $\bullet$ \\
\hline 7779 & & $\bullet$ & \\
\hline 7788 & & $\bullet$ & \\
\hline 7792 & & $\bullet$ & \\
\hline 7838 & & $\bullet$ & \\
\hline 7839 & & $\bullet$ & $\bullet$ \\
\hline 7851 & & $\bullet$ & \\
\hline 7855 & & $\bullet$ & \\
\hline 7860 & & $\bullet$ & \\
\hline 7866 & & $\bullet$ & \\
\hline 7869 & & $\bullet$ & $\bullet$ \\
\hline 7872 & & $\bullet$ & \\
\hline 7926 & & $\bullet$ & \\
\hline 7930 & & $\bullet$ & \\
\hline 7938 & & $\bullet$ & \\
\hline 7950 & & $\bullet$ & \\
\hline 7963 & & $\bullet$ & \\
\hline 7980 & & $\bullet$ & \\
\hline 7982 & & $\bullet$ & \\
\hline 7988 & & $\bullet$ & \\
\hline 7996 & & $\bullet$ & \\
\hline 7999 & & $\bullet$ & $\bullet$ \\
\hline 8001 & & & $\bullet$ \\
\hline
\end{tabular}




\begin{tabular}{|c|c|c|c|c|}
\hline Nr. & $\stackrel{\mathscr{D}}{0}$ & $\begin{array}{l}N \\
\cdot \vec{\Xi} \\
\stackrel{D}{ } \\
\tilde{N}\end{array}$ & $\begin{array}{l}\vec{H} \\
\cdot \vec{\Xi} \\
\otimes \\
ٌ\end{array}$ & $\frac{\tilde{0}}{\frac{\pi}{0}}$ \\
\hline 8016 & & & $\bullet$ & \\
\hline 8017 & & $\bullet$ & & \\
\hline 8024 & & $\bullet$ & & \\
\hline 8030 & & $\bullet$ & & \\
\hline 8032 & & $\bullet$ & & \\
\hline 8036 & & & $\bullet$ & \\
\hline 8039 & & $\bullet$ & & \\
\hline 8040 & & $\bullet$ & & \\
\hline 8041 & & & $\bullet$ & \\
\hline 8044 & & & $\bullet$ & \\
\hline 8048 & & $\bullet$ & & \\
\hline 8063 & & $\bullet$ & & \\
\hline 8073 & & $\bullet$ & & \\
\hline 8074 & & $\bullet$ & & \\
\hline 8077 & & $\bullet$ & $\bullet$ & \\
\hline 8082 & & $\bullet$ & $\bullet$ & \\
\hline 8084 & & $\bullet$ & & \\
\hline 8085 & & $\bullet$ & $\bullet$ & \\
\hline 8086 & & $\bullet$ & $\bullet$ & \\
\hline 8087 & & $\bullet$ & & \\
\hline 8092 & & $\bullet$ & & \\
\hline 8096 & & $\bullet$ & $\bullet$ & \\
\hline 8104 & & & $\bullet$ & \\
\hline 8107 & & $\bullet$ & $\bullet$ & \\
\hline 8109 & & $\bullet$ & 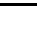 & \\
\hline 8116 & & $\bullet$ & $\bullet$ & \\
\hline 8121 & & $\bullet$ & & \\
\hline 8131 & & $\bullet$ & & \\
\hline 8134 & & $\bullet$ & & \\
\hline 8152 & & $\bullet$ & & \\
\hline 8154 & & $\bullet$ & & \\
\hline 8172 & & $\bullet$ & & \\
\hline 8179 & & & $\bullet$ & \\
\hline 8181 & & $\bullet$ & & \\
\hline 8198 & & $\bullet$ & & \\
\hline 8227 & & $\bullet$ & & \\
\hline 8239 & & $\bullet$ & $\bullet$ & \\
\hline 8241 & & $\bullet$ & $\bullet$ & \\
\hline 8244 & & $\bullet$ & & \\
\hline 8306 & & $\bullet$ & & \\
\hline 8335 & & $\bullet$ & & \\
\hline 8337 & & $\bullet$ & & \\
\hline
\end{tabular}

\begin{tabular}{|c|c|c|c|c|}
\hline Nr. & $\stackrel{v}{0}$ & 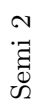 & $\begin{array}{l}+ \\
\cdot \vec{\Xi} \\
\varpi \\
\tilde{D}\end{array}$ & $\mid \begin{array}{l}\frac{1}{0} \\
\frac{1}{13} \\
\frac{\pi}{2} \\
\sum\end{array}$ \\
\hline 8348 & & $\bullet$ & & \\
\hline 8355 & & $\bullet$ & & \\
\hline 8362 & & $\bullet$ & & \\
\hline 8370 & & $\bullet$ & & \\
\hline 8402 & & $\bullet$ & & \\
\hline 8423 & & $\bullet$ & & \\
\hline 8425 & $\bullet$ & $\bullet$ & & \\
\hline 8488 & & $\bullet$ & & \\
\hline 8491 & & $\bullet$ & & \\
\hline 8498 & & $\bullet$ & & \\
\hline 8509 & & $\bullet$ & & \\
\hline 8518 & & - & & \\
\hline 8521 & & $\bullet$ & & \\
\hline 8524 & & $\bullet$ & & \\
\hline 8530 & & $\bullet$ & & \\
\hline 8546 & & $\bullet$ & & \\
\hline 8561 & & $\bullet$ & & \\
\hline 8562 & & $\bullet$ & & \\
\hline 8569 & & $\bullet$ & & \\
\hline 8584 & & $\bullet$ & & \\
\hline 8589 & & $\bullet$ & & \\
\hline 8651 & & $\bullet$ & & \\
\hline 8671 & & $\bullet$ & & \\
\hline 8673 & & $\bullet$ & & \\
\hline 8679 & & $\bullet$ & $\bullet$ & \\
\hline 8680 & & $\bullet$ & & \\
\hline 8686 & & $\bullet$ & & \\
\hline 8687 & & $\bullet$ & $\bullet$ & \\
\hline 8691 & & $\bullet$ & & \\
\hline 8695 & & $\bullet$ & & \\
\hline 8708 & & $\bullet$ & & \\
\hline 8712 & & $\bullet$ & & \\
\hline 8717 & & $\bullet$ & & \\
\hline 8728 & & $\bullet$ & & \\
\hline 8735 & & $\bullet$ & & \\
\hline 8740 & & $\bullet$ & & \\
\hline 8748 & & $\bullet$ & & \\
\hline 8750 & & $\bullet$ & $\bullet$ & \\
\hline 8754 & & $\bullet$ & & \\
\hline 8755 & & $\bullet$ & & \\
\hline 8766 & & $\bullet$ & & \\
\hline 8767 & & $\bullet$ & & \\
\hline
\end{tabular}

\begin{tabular}{|c|c|c|c|c|}
\hline Nr. & $\stackrel{\mathscr{D}}{\oplus}$ & 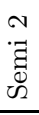 & $\begin{array}{l}+ \\
\vec{\Xi} \\
\tilde{\nu} \\
\tilde{~}\end{array}$ & 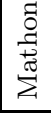 \\
\hline 8778 & & $\bullet$ & & \\
\hline 8781 & & • & & \\
\hline 8782 & & & $\bullet$ & \\
\hline 8787 & & $\bullet$ & & \\
\hline 8801 & & $\bullet$ & & \\
\hline 8805 & & $\bullet$ & & \\
\hline 8835 & & $\bullet$ & & \\
\hline 8837 & $\bullet$ & $\bullet$ & & \\
\hline 8842 & & $\bullet$ & $\bullet$ & \\
\hline 8845 & & $\bullet$ & $\bullet$ & \\
\hline 8846 & & $\bullet$ & $\bullet$ & \\
\hline 8848 & & $\bullet$ & & \\
\hline 8849 & & $\bullet$ & & \\
\hline 8850 & & $\bullet$ & & \\
\hline 8855 & & $\bullet$ & & \\
\hline 8860 & & $\bullet$ & & \\
\hline 8875 & & $\bullet$ & & \\
\hline 8876 & & $\bullet$ & & \\
\hline 8878 & & $\bullet$ & & \\
\hline 8879 & & $\bullet$ & & \\
\hline 8884 & & & $\bullet$ & \\
\hline 8891 & & $\bullet$ & & \\
\hline 8906 & & $\bullet$ & & \\
\hline 8921 & & $\bullet$ & & \\
\hline 8923 & & 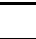 & $\bullet$ & \\
\hline 8925 & & $\bullet$ & & \\
\hline 8930 & & $\bullet$ & & \\
\hline 8952 & & $\bullet$ & & \\
\hline 8987 & & $\bullet$ & & \\
\hline 8989 & & $\bullet$ & & \\
\hline 8991 & $\bullet$ & & & \\
\hline 8995 & & $\bullet$ & & \\
\hline 8997 & & $\bullet$ & & \\
\hline 9010 & $\bullet$ & . & & \\
\hline 9019 & & $\bullet$ & & \\
\hline 9021 & & $\bullet$ & & \\
\hline 9025 & & $\bullet$ & & \\
\hline 9028 & & $\bullet$ & & \\
\hline 9029 & & $\bullet$ & & \\
\hline 9046 & & $\bullet$ & & \\
\hline 9051 & & $\bullet$ & $\bullet$ & \\
\hline 9053 & & $\bullet$ & & \\
\hline
\end{tabular}


M. RÖDER

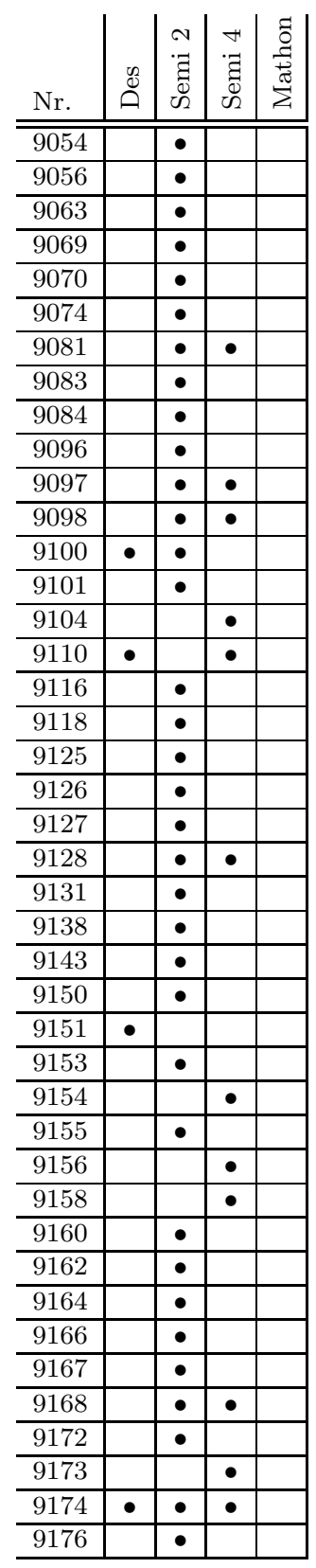

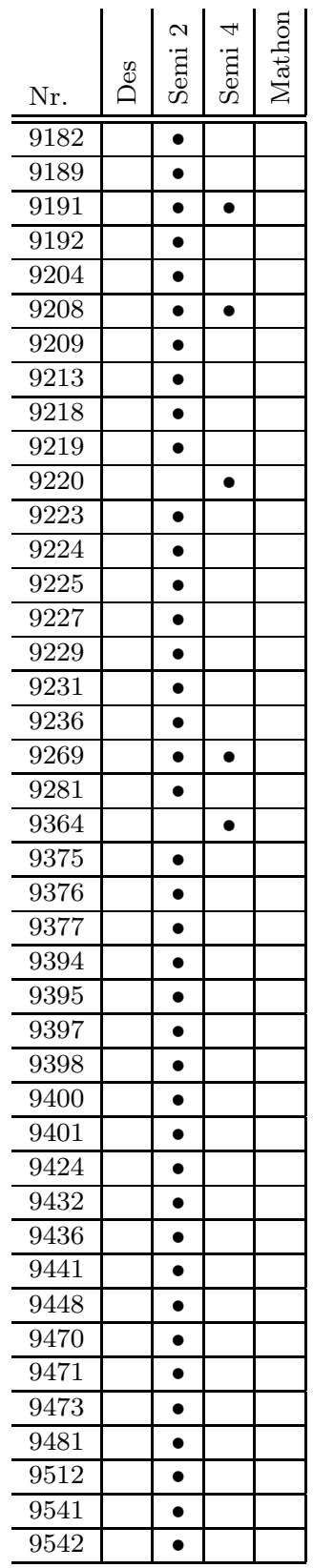

\begin{tabular}{|c|c|c|c|c|}
\hline Nr. & $\stackrel{\infty}{0}$ & $\begin{array}{c}N \\
\cdot \vec{\Xi} \\
\tilde{D} \\
\tilde{D}\end{array}$ & 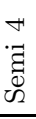 & 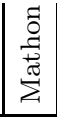 \\
\hline$\overline{9553}$ & & • & & \\
\hline 9558 & & $\bullet$ & & \\
\hline 9564 & & $\bullet$ & & \\
\hline 9582 & & $\bullet$ & & \\
\hline 9586 & & $\bullet$ & & \\
\hline 9598 & & & $\bullet$ & \\
\hline 9599 & & $\bullet$ & & \\
\hline 9613 & & $\bullet$ & & \\
\hline 9617 & & $\bullet$ & $\bullet$ & \\
\hline $\begin{array}{l}9618 \\
\end{array}$ & & $\bullet$ & $\bullet$ & \\
\hline $\begin{array}{l}9674 \\
\end{array}$ & & $\bullet$ & $\bullet$ & \\
\hline $\begin{array}{l}9675 \\
\end{array}$ & & $\bullet$ & & \\
\hline 9676 & & $\bullet$ & & \\
\hline 9683 & & $\bullet$ & & \\
\hline 9697 & & $\bullet$ & & \\
\hline 9698 & & $\bullet$ & & \\
\hline 9699 & & $\bullet$ & & \\
\hline 9701 & & $\bullet$ & & \\
\hline 9704 & & $\bullet$ & & \\
\hline 9709 & & $\bullet$ & & \\
\hline 9714 & & $\bullet$ & & \\
\hline 9720 & & $\bullet$ & & \\
\hline 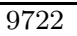 & & $\bullet$ & & \\
\hline 9727 & & $\bullet$ & & \\
\hline 9732 & & $\bullet$ & & \\
\hline 9733 & & & $\bullet$ & \\
\hline 9738 & & $\bullet$ & & \\
\hline 9740 & & & $\bullet$ & \\
\hline 9745 & & $\bullet$ & & \\
\hline 9748 & & $\bullet$ & & \\
\hline 9754 & & $\bullet$ & & \\
\hline 9757 & & $\bullet$ & & \\
\hline $\begin{array}{l}758 \\
\end{array}$ & & $\bullet$ & & \\
\hline 9764 & & $\bullet$ & & \\
\hline 9771 & & $\bullet$ & & \\
\hline 9772 & & $\bullet$ & & \\
\hline 9778 & & & $\bullet$ & \\
\hline 9780 & & $\bullet$ & & \\
\hline 9784 & & $\bullet$ & & \\
\hline 9794 & & $\bullet$ & & \\
\hline 9801 & & $\bullet$ & & \\
\hline 9814 & & $\bullet$ & & \\
\hline
\end{tabular}




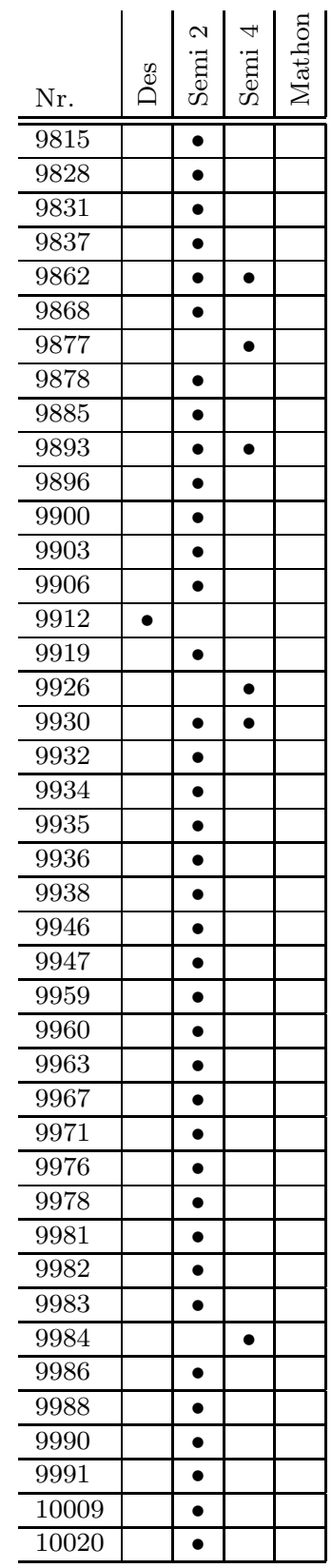

\begin{tabular}{|c|c|c|c|c|}
\hline Nr. & $\stackrel{\tilde{D}^{2}}{0}$ & 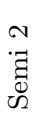 & $\begin{array}{l}F \\
\overrightarrow{\text { J }} \\
\text { W }\end{array}$ & 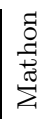 \\
\hline 10022 & & $\bullet$ & & \\
\hline 10024 & & $\bullet$ & & \\
\hline 10030 & & $\bullet$ & & \\
\hline 10039 & & & $\bullet$ & \\
\hline 10041 & & $\bullet$ & $\bullet$ & \\
\hline 10042 & & $\bullet$ & • & \\
\hline 10043 & & $\bullet$ & & \\
\hline 10060 & & $\bullet$ & $\bullet$ & \\
\hline 10066 & $\bullet$ & & & \\
\hline 10069 & & $\bullet$ & & \\
\hline 10073 & & $\bullet$ & & \\
\hline 10100 & & & $\bullet$ & \\
\hline 10116 & & $\bullet$ & & \\
\hline 10120 & & $\bullet$ & & \\
\hline 10142 & & $\bullet$ & & \\
\hline 10150 & & $\bullet$ & & \\
\hline 10166 & & $\bullet$ & & \\
\hline 10173 & & $\bullet$ & $\bullet$ & \\
\hline 10179 & & $\bullet$ & & \\
\hline 10190 & & $\bullet$ & & \\
\hline 10197 & & $\bullet$ & & \\
\hline 10198 & & & $\bullet$ & \\
\hline 10200 & & $\bullet$ & & \\
\hline 10206 & & $\bullet$ & & \\
\hline 10207 & & $\bullet$ & & \\
\hline 10234 & & & $\bullet$ & \\
\hline 10244 & & & $\bullet$ & \\
\hline 10246 & & $\bullet$ & & \\
\hline 10254 & & $\bullet$ & & \\
\hline 10263 & & $\bullet$ & & \\
\hline 10266 & & $\bullet$ & & \\
\hline 10268 & & & $\bullet$ & \\
\hline 10269 & & $\bullet$ & $\bullet$ & \\
\hline 10272 & & $\bullet$ & $\bullet$ & \\
\hline 10277 & & $\bullet$ & $\bullet$ & \\
\hline 10283 & & $\bullet$ & & \\
\hline 10285 & & $\bullet$ & & \\
\hline 10287 & & $\bullet$ & & \\
\hline 10294 & & $\bullet$ & & \\
\hline 10295 & & $\bullet$ & $\bullet$ & \\
\hline 10296 & & $\bullet$ & & \\
\hline 10297 & $\bullet$ & $\bullet$ & $\bullet$ & \\
\hline 10313 & & & & $\bullet$ \\
\hline
\end{tabular}

\begin{tabular}{|c|c|c|c|c|}
\hline Nr. & $\stackrel{\mathscr{D}}{0}$ & $\begin{array}{l}\text { N } \\
\stackrel{\vec{g}}{\varpi} \\
\tilde{N}\end{array}$ & 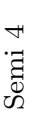 & 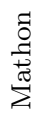 \\
\hline 10437 & & $\bullet$ & $\bullet$ & \\
\hline 10528 & & & & $\bullet$ \\
\hline 10572 & & & & $\bullet$ \\
\hline 10636 & & & & $\bullet$ \\
\hline 10655 & $\bullet$ & $\bullet$ & & \\
\hline 10730 & & $\bullet$ & & \\
\hline 10734 & & & $\bullet$ & $\bullet$ \\
\hline 10739 & & & & $\bullet$ \\
\hline 10785 & & & & $\bullet$ \\
\hline 10796 & & & & $\bullet$ \\
\hline 10808 & & & & $\bullet$ \\
\hline 13317 & & & & $\bullet$ \\
\hline 13780 & & & & $\bullet$ \\
\hline 14204 & & & & $\bullet$ \\
\hline 14819 & & & & $\bullet$ \\
\hline 14829 & & & & $\bullet$ \\
\hline 27067 & & $\bullet$ & & \\
\hline 27101 & & $\bullet$ & & \\
\hline 27106 & & $\bullet$ & & \\
\hline 27131 & & $\bullet$ & & \\
\hline 27333 & & $\bullet$ & $\bullet$ & \\
\hline 27534 & & $\bullet$ & $\bullet$ & \\
\hline 27588 & & $\bullet$ & & \\
\hline 27677 & & $\bullet$ & & \\
\hline 27848 & & & $\bullet$ & \\
\hline 27880 & & $\bullet$ & & \\
\hline 27887 & & $\bullet$ & & \\
\hline 27916 & & $\bullet$ & & \\
\hline 27928 & & $\bullet$ & & \\
\hline 27932 & & & $\bullet$ & \\
\hline 29622 & $\bullet$ & & & \\
\hline 29676 & & $\bullet$ & & \\
\hline 29677 & & $\bullet$ & & \\
\hline 45194 & & $\bullet$ & & \\
\hline 45224 & & $\bullet$ & & \\
\hline 45244 & & $\bullet$ & & \\
\hline 45253 & & $\bullet$ & & \\
\hline 45257 & & $\bullet$ & & \\
\hline 45259 & & $\bullet$ & $\bullet$ & \\
\hline 45274 & $\bullet$ & $\bullet$ & $\bullet$ & \\
\hline 53237 & & & & $\bullet$ \\
\hline 53830 & & & & $\bullet$ \\
\hline 53959 & & & & $\bullet$ \\
\hline
\end{tabular}




\section{REFERENCES}

[Bru55] R. H. Bruck, Difference sets in a finite group, Trans. Amer. Math. Soc. 78 (1955), 464-481.

[Dem68] P. Dembowski, Finite geometries, Ergebnisse der Mathematik und ihrer Grenzgebiete 44, Springer-Verlag, Berlin-Heidelberg, 1968.

[DP67] P. Dembowski and F. Piper, Quasiregular collineation groups of finite projective planes, Math. Z. 99 (1967), 53-75.

[DR83] U. Dempwolff and A. Reifart, The classification of the translation planes of order 16. I, Geom. Dedicata 15 (1983), 137-153.

[GAP] The GAP Group, GAP - Groups, Algorithms, and Programming, Version 4.4, http://www.gap-system.org.

[GJ03] D. Ghinelli and D. Jungnickel, On finite projective planes in Lenz-Barlotti class at least I.3, Adv. Geom. Special Issue (2003), S28-S48.

[Pot95] A. Pott, Finite geometry and character theory, Lecture Notes in Mathematics 1601, Springer Verlag, Berlin Heidelberg, 1995.

[Röd] M. Röder, http://www.maths.nuigalway.ie/ roeder.

[Röd06a] M. Röder, Quasiregular projective planes of order 16 - a computational approach, Ph.D. thesis, Technische Universität Kaiserslautern, 2006, http://kluedo.ub. uni-kl.de/volltexte/2006/2036/.

[Röd06b] M. Röder, RDS - a GAP4 package for relative difference sets, 2006, http://www . gap-system.org/Packages/rds.html.

[Roy] G. Royle, Gordon Royle's planes of order 16, http://www.csse.uwa.edu.au/ gordon/remote/planes16/index.html.

[Sch02] B. Schmidt, Characters and cyclotomic fields in finite geometry, Lecture Notes in Mathematics 1797, Springer-Verlag, Berlin, 2002.

M. Röder

Department of Mathematics

National University of Ireland

University Road

Galway

Ireland

E-mail: marc.roeder@nuigalway.ie

Received: 24.1.2008.

Revised: 28.3.2008. 